\title{
The extragalactic background light revisited and the cosmic photon-photon opacity (Corrigendum)
}

\author{
Alberto Franceschini and Giulia Rodighiero \\ University of Padova, Physics and Astronomy Department, Vicolo Osservatorio 3, 35122 Padova, Italy \\ e-mail: alberto.franceschini@unipd.it
}

A\&A, 603, A34 (2017), https://doi.org/10.1051/0004-6361/201629684

Key words. cosmic background radiation - diffuse radiation - gamma rays: galaxies - BL Lacertae objects: general - errata, addenda

For some unexplained reasons, all tables in the Franceschini \& Rodighiero (2017) paper have been mixed up. Tables $1-3$ reported there were identical to those published in Franceschini et al. (2008). We report here in Tables 1, 2, and 4 the corresponding correct values in terms of photon proper number densities and photon-photon optical depths as a function of photon energy.

We also took this occasion to add a new table (Table 3) including the predicted extragalactic background light values at redshifts between 2 and 3.5, as requested by some readers of the Franceschini \& Rodighiero (2017) paper.

Both photon number densities and photon-photon optical depths are calculated including the contributions of the cosmic microwave background assumed as a black-body with $T=2.728 \mathrm{~K}$. Except for the numerical values in the tables, all the rest of the Franceschini \& Rodighiero (2017) paper is unaffected by the problem.

Acknowledgements. We thank Jonathan Biteau, Barbara Biasuzzi, and Christoph Wendel for useful comments and criticisms.

\section{References}

Franceschini, A., \& Rodighiero, G. 2017, A\&A 603, A34

Franceschini, A., Rodighiero, G., \& Vaccari, M. 2008, A\&A, 487, 837 
Table 1. Photon proper number density as a function of redshift (1).

\begin{tabular}{|c|c|c|c|c|c|c|c|c|c|c|c|}
\hline \multicolumn{2}{|c|}{$z=0$} & \multicolumn{2}{|c|}{$z=0.2$} & \multicolumn{2}{|c|}{$z=0.4$} & \multicolumn{2}{|c|}{$z=0.6$} & \multicolumn{2}{|c|}{$z=0.8$} & \multicolumn{2}{|c|}{$z=1.0$} \\
\hline $\log \epsilon^{a}$ & $\log \left(\epsilon \frac{\mathrm{d} n_{\gamma}}{\mathrm{d} \epsilon}\right)^{b}$ & $\log \epsilon$ & $\log \left(\epsilon \frac{\mathrm{d} n_{\gamma}}{\mathrm{d} \epsilon}\right)$ & $\log \epsilon$ & $\log \left(\epsilon \frac{\mathrm{d} n_{\gamma}}{\mathrm{d} \epsilon}\right)$ & $\log \epsilon$ & $\log \left(\epsilon \frac{\mathrm{d} n_{\gamma}}{\mathrm{d} \epsilon}\right)$ & $\log \epsilon$ & $\log \left(\epsilon \frac{\mathrm{d} n_{\gamma}}{\mathrm{d} \epsilon}\right)$ & $\log \epsilon$ & $\log \left(\epsilon \frac{\mathrm{d} n_{\gamma}}{\mathrm{d} \epsilon}\right)$ \\
\hline-2.8834 & 2.0525 & -2.8042 & 2.2108 & -2.7873 & 2.4598 & -2.7294 & 2.5758 & -2.6782 & 2.6781 & -2.6325 & 2.7695 \\
\hline & 1.9065 & -2.7542 & 2.0648 & -2.7372 & 2.3447 & -2.6793 & 2.4607 & -2.6281 & 2.5630 & -2.5824 & 2.6544 \\
\hline-2.7834 & 1.7255 & -2.7042 & 1.8838 & -2.6872 & 2.1987 & -2.6293 & 2.3147 & -2.5781 & 2.4170 & -2.5324 & 2.5084 \\
\hline-2.7334 & 1.5035 & -2.6542 & 1.6618 & -2.6372 & 2.0177 & -2.5793 & 2.1337 & -2.5281 & 2.2360 & -2.4824 & 2.3274 \\
\hline-2.6834 & 1.2365 & -2.6042 & 1.3948 & -2.5872 & 1.7957 & -2.5293 & 1.9117 & -2.4781 & 2.0140 & -2.4324 & 2.1054 \\
\hline-2.6334 & 0.9185 & -2.5542 & 1.0768 & -2.5372 & 1.5287 & -2.4793 & 1.6447 & -2.4281 & 1.7470 & -2.3824 & 1.8384 \\
\hline-2.5834 & 0.5435 & -2.5042 & 0.7018 & -2.4872 & 1.2107 & -2.4293 & 1.3267 & -2.3781 & 1.4290 & -2.3324 & 1.5204 \\
\hline-2.5334 & 0.1839 & -2.4542 & 0.3553 & -2.4372 & 0.8694 & -2.3793 & 0.9885 & -2.3281 & 1.0922 & -2.2824 & 1.1834 \\
\hline-2.4495 & -0.2972 & -2.3703 & -0.0893 & -2.3034 & 0.0803 & -2.2454 & 0.2179 & -2.1942 & 0.3140 & -2.1485 & 0.3790 \\
\hline-2.3034 & -0.3062 & -2.2242 & -0.0961 & -2.1573 & 0.0722 & -2.0993 & 0.2005 & -2.0481 & 0.2751 & -2.0024 & 0.3130 \\
\hline-2.1359 & -0.2983 & -2.0567 & -0.1042 & -1.9897 & 0.0584 & -1.9318 & 0.1752 & -1.8807 & 0.2245 & -1.8348 & 0.2330 \\
\hline-2.0516 & -0.3699 & -1.9722 & -0.1809 & -1.9055 & -0.0251 & -1.8474 & 0.0803 & -1.7964 & 0.1126 & -1.7506 & 0.1011 \\
\hline-1 & -0 & -1.8677 & -0.2979 & -1.8008 & -0.1489 & -1.7428 & -0.0542 & -1.6916 & -0.0391 & -1.6459 & -0.0719 \\
\hline-1.8598 & -0.7073 & -1.7804 & -0.5333 & -1.7135 & -0.3934 & -1.6556 & -0.3186 & -1.6045 & -0.3227 & -1.5586 & -0.3554 \\
\hline-1.7804 & -0.8955 & -1.7014 & -0.7282 & -1.6343 & -0.5962 & -1.5764 & -0.5232 & -1.5252 & -0.5245 & -1.4795 & -0.5541 \\
\hline-1.7506 & -0.9674 & -1.6714 & -0.7981 & -1.6045 & -0.6645 & -1.5464 & -0.5875 & -1.4953 & -0.5834 & -1.4495 & -0.6068 \\
\hline-1.6836 & -1.1025 & -1.6045 & -0.9374 & -1.5375 & -0.8060 & -1.4795 & -0.7307 & -1.4283 & -0.7284 & -1.3826 & -0.7542 \\
\hline & -1.5699 & -1.4 & -1.3836 & -1.3614 & -1.2438 & -1.3034 & -1.1540 & -1.2523 & -1.1288 & -1.2065 & -1.1364 \\
\hline-1 & -1.7910 & -1.3 & -1.6185 & -1.2364 & -1.4717 & -1.1785 & -1.3804 & -1.1273 & -1.3503 & -1.0816 & -1.3479 \\
\hline-1.2857 & -1.8824 & -1.2065 & -1.6641 & -1.1395 & -1.5053 & -1.0816 & -1.3971 & -1.0304 & -1.3490 & -0.9846 & -1.3545 \\
\hline-1.2065 & -1.9245 & -1.1273 & -1.7807 & -1.0603 & -1.6245 & -1.0024 & -1.5209 & -0.9512 & -1.4603 & -0.9055 & -1.3982 \\
\hline-1.0816 & -2.1192 & -1.0024 & -1.9363 & -0.9355 & -1.7881 & -0.8775 & -1.6686 & -0.8262 & -1.6293 & -0.7804 & -1.6501 \\
\hline-0 & -2.2 & -0.9055 & -2.1152 & -0.8386 & -1.9893 & -0.7804 & -1.9 & -0.7293 & -1.9289 & -0.6836 & -1.9594 \\
\hline-0.8598 & -2.4631 & -0.7804 & -2.3049 & -0.7135 & -2.1725 & -0.6556 & -2.0848 & -0.6045 & -2.0699 & -0.5586 & -2.0711 \\
\hline-0.8085 & -2.5029 & -0.7293 & -2.3370 & -0.6623 & -2.2012 & -0.6045 & -2.1087 & -0.5533 & -2.0785 & -0.5075 & -2.0669 \\
\hline-0.7314 & -2.4740 & -0.6523 & -2.2961 & -0.5854 & -2.1549 & -0.5274 & -2.0548 & -0.4763 & -2.0026 & -0.4305 & -1.9741 \\
\hline-0.6688 & -2.4869 & -0.5897 & -2.2986 & -0.5227 & -2.1519 & -0.4647 & -2.0 & -0.4136 & -1.9904 & -0.3678 & -1.9532 \\
\hline-0.5597 & -2.4444 & -0.4805 & -2.2465 & -0.4136 & -2.1010 & -0.3556 & -1.9960 & -0.3045 & -1.9235 & -0.2587 & -1.8871 \\
\hline-0.4713 & -2.3871 & -0.3921 & -2.1981 & -0.3252 & -2.0522 & -0.2672 & -1.9536 & -0.2160 & -1.9020 & -0.1703 & -1.8887 \\
\hline-0.3792 & -2.3534 & -0.300 & -2.1624 & -0.2331 & -2.0327 & -0.1751 & -1.9582 & -0.1240 & -1.9311 & -0.0783 & -1.9409 \\
\hline-0 & -2.3635 & -0.2373 & -2.1870 & -0.1704 & -2.0680 & -0.1124 & -2.0018 & -0.0612 & -1.9804 & -0.0155 & -1.9935 \\
\hline-0.2796 & -2.3694 & -02004 & -2.2014 & -0.1335 & -2.0888 & -0.0755 & -2.0274 & -0.0244 & -2.0093 & 0.0214 & -2.0244 \\
\hline-0.2649 & -2.3718 & -0.1857 & -2.2072 & -0.1188 & -2.0970 & -0.0608 & -2.0376 & -0.0097 & -2.0208 & 0.0361 & -2.0367 \\
\hline-0.2 & -2.3748 & -0 & -2.2144 & -0.1004 & -2.1074 & -0.0424 & -2.0 & 0.0088 & -2.0353 & 0.0545 & -2.0522 \\
\hline-0.2132 & -2.3801 & -01340 & -2.2274 & -0.0671 & -2.1261 & -0.0091 & -20736 & 0.0420 & -2.0614 & 00878 & -2.0800 \\
\hline-0.1910 & -2.3837 & -0.1118 & -2.2361 & -0.0449 & -2.1386 & 0.0131 & -2.0890 & 0.0642 & -2.0788 & 0.1100 & -2.0986 \\
\hline-0.1651 & -2.3878 & -0.0859 & -2.2463 & -0.0190 & -2.1532 & 0.0390 & -2.1070 & 0.0901 & -2.0991 & 0.1359 & -2.1204 \\
\hline-0.1429 & -2.3914 & -0.0638 & -2.2550 & 0.0032 & -2.1657 & 0.0612 & -2.1225 & 0.1123 & -2.1165 & 0.1581 & -2.1389 \\
\hline-0.1207 & -2.4066 & -0.0415 & -2.2723 & 0.0254 & -2.1869 & 0.0834 & -2.1459 & 0.1345 & -2.1402 & 0.1803 & -2.1624 \\
\hline-0.0873 & -2.4374 & -0.0081 & -2.3046 & 0.0588 & -2.2248 & 0.1168 & -2.1866 & 01670 & -2.1800 & 0.2137 & -2.2013 \\
\hline-0.0687 & -2.4546 & 0.0105 & -2.3225 & 0.0774 & -2.2459 & 0.1354 & -2.2093 & 0.1865 & -2.2021 & 0.2323 & -2.2229 \\
\hline-0.0575 & -2.4650 & 0.0217 & -2.3333 & 0.0886 & -2.2587 & 0.1466 & -2.2230 & 0.1977 & -2.2154 & 0.2435 & -2.2359 \\
\hline-0.0461 & -2.4755 & 0.0331 & -2.3443 & 0.1000 & -2.2716 & 0.1580 & -2.2369 & 0.2091 & -2.2290 & 0.2549 & -2.2492 \\
\hline-0.0272 & -2.4930 & 0.0520 & -2.3625 & 0.1189 & -2.2931 & 0.1769 & -2.2599 & 0.2280 & -2.2515 & 0.2738 & -2.2712 \\
\hline-0.0083 & -2.5141 & 0.0709 & -2.3867 & 0.1378 & -2.3190 & 0.1958 & -2.2870 & 0.2469 & -2.2803 & 0.2927 & -2.3027 \\
\hline 0.0907 & -2.6383 & 0.1698 & -2.5363 & 0.2368 & -2.4711 & 0.2948 & -2.4432 & 0.3459 & -2.4534 & 0.3917 & -2.5034 \\
\hline 0.0945 & -2.6431 & 0.1738 & -2.5422 & 0.2405 & -2.4768 & 0.2986 & -2.4493 & 0.3499 & -2.4603 & 0.3955 & -2.5113 \\
\hline & -2.8281 & 0.2707 & -2.7254 & 0.3377 & -2.6818 & 0.3955 & -2.6859 & 0.4467 & -2.7347 & 0.4925 & -2.8093 \\
\hline 0.3541 & -3.1463 & 0.4334 & -3.1134 & 0.5004 & -3.1043 & 0.5583 & -3.0633 & 0.6095 & -3.0197 & 0.6552 & -2.9703 \\
\hline 0.4925 & -3.5493 & 0.5717 & -3.4470 & 0.6386 & -3.3511 & 0.6966 & -3.2464 & 0.7477 & -3.1553 & 0.7935 & -3.0846 \\
\hline 0.7935 & -4.1744 & 0.8727 & -4.0370 & 0.9396 & -3.9704 & 0.9976 & -3.9531 & 1.0488 & -3.9330 & 1.0945 & -3.8875 \\
\hline
\end{tabular}

Notes. ${ }^{(a)}$ Photon energies $\epsilon$ are in $\mathrm{eV} .{ }^{(b)}$ Photon densities $\epsilon \frac{\mathrm{d} n_{\gamma}}{\mathrm{d} \epsilon}$ are in $\left[\mathrm{cm}^{-3}\right]$. 
Table 2. Photon proper number density as a function of redshift (2).

\begin{tabular}{|c|c|c|c|c|c|c|c|c|c|}
\hline \multicolumn{2}{|c|}{$z=1.2$} & \multicolumn{2}{|c|}{$z=1.4$} & \multicolumn{2}{|c|}{$z=1.6$} & \multicolumn{2}{|c|}{$z=1.8$} & \multicolumn{2}{|c|}{$z=2.0$} \\
\hline $\log \epsilon^{a}$ & $\log \left(\epsilon \frac{\mathrm{d} n_{\gamma}}{\mathrm{d} \epsilon}\right)^{b}$ & $\log \epsilon$ & $\log \left(\epsilon \frac{\mathrm{d} n_{\gamma}}{\mathrm{d} \epsilon}\right)$ & $\log \epsilon$ & $\log \left(\epsilon \frac{\mathrm{d} n_{\gamma}}{\mathrm{d} \epsilon}\right)$ & $\log \epsilon$ & $\log \left(\epsilon \frac{\mathrm{d} n_{\gamma}}{\mathrm{d} \epsilon}\right)$ & $\log \epsilon$ & $\log \left(\epsilon \frac{\mathrm{d} n_{\gamma}}{\mathrm{d} \epsilon}\right)$ \\
\hline-2.5410 & 2.7372 & -2.5032 & 2.8128 & -2.4684 & 2.8823 & -2.4362 & 2.9467 & -2.4063 & 3.0066 \\
\hline-2.4910 & 2.5912 & -2.4532 & 2.6668 & -2.4184 & 2.7363 & -2.3862 & 2.8007 & -2.3563 & 2.8606 \\
\hline-2.4410 & 2.4102 & -2.4032 & 2.4858 & -2.3684 & 2.5553 & -2.3362 & 2.6197 & -2.3063 & 2.6796 \\
\hline-2.3910 & 2.1882 & -2.3532 & 2.2638 & -2.3184 & 2.3333 & -2.2862 & 2.3977 & -2.2563 & 2.4576 \\
\hline-2.3410 & 1.9212 & -2.3032 & 1.9968 & -2.2684 & 2.0663 & -2.2362 & 2.1307 & -2.2063 & 2.1906 \\
\hline-2.2910 & 1.6032 & -2.2532 & 1.6788 & -2.2184 & 1.7483 & -2.1862 & 1.8127 & -2.1563 & 1.8726 \\
\hline-2.2410 & 1.2282 & -2.2032 & 1.3038 & -2.1684 & 1.3733 & -2.1362 & 1.4377 & -2.1063 & 1.4976 \\
\hline-2.1910 & 0.8942 & -2.1532 & 0.9615 & -2.1184 & 1.0215 & -2.0862 & 1.0765 & -2.0563 & 1.1277 \\
\hline-2.1071 & 0.4363 & -2.0693 & 0.4732 & -2.0346 & 0.5020 & -2.0024 & 0.5253 & -1.9722 & 0.5449 \\
\hline-1.9610 & 0.3406 & -1.9234 & 0.3432 & -1.8884 & 0.3310 & -1.8564 & 0.3085 & -1.8262 & 0.2765 \\
\hline-1.7934 & 0.2180 & -1.7557 & 0.1770 & -1.7210 & 0.1248 & -1.6887 & 0.0607 & -1.6588 & -0.0101 \\
\hline-1.7091 & 0.0667 & -1.6714 & 0.0022 & -1.6366 & -0.0682 & -1.6045 & -0.1385 & -1.5745 & -0.2066 \\
\hline-1.6045 & -0.1226 & -1.5667 & -0.1886 & -1.5319 & -0.2548 & -1.4998 & -0.3188 & -1.4697 & -0.3806 \\
\hline-1.5173 & -0.3989 & -1.4795 & -0.4578 & -1.4448 & -0.5186 & -1.4125 & -0.5822 & -1.3826 & -0.6505 \\
\hline-1.4381 & -0.5950 & -1.4003 & -0.6552 & -1.3655 & -0.7196 & -1.3334 & -0.7844 & -1.3034 & -0.8508 \\
\hline-1.4082 & -0.6411 & -1.3704 & -0.6938 & -1.3356 & -0.7486 & -1.3034 & -0.8022 & -1.2734 & -0.8542 \\
\hline-1.3412 & -0.7916 & -1.3034 & -0.8468 & -1.2687 & -0.9020 & -1.2364 & -0.9582 & -1.2065 & -1.0175 \\
\hline-1.1651 & -1.1519 & -1.1273 & -1.1756 & -1.0925 & -1.1974 & -1.0604 & -1.2007 & -1.0304 & -1.2146 \\
\hline-1.0401 & -1.3461 & -1.0024 & -1.3801 & -0.9678 & -1.4397 & -0.9355 & -1.4734 & -0.9055 & -1.4750 \\
\hline-0.9431 & -1.3512 & -0.9055 & -1.3205 & -0.8706 & -1.2847 & -0.8386 & -1.2890 & -0.8085 & -1.3492 \\
\hline-0.8642 & -1.3601 & -0.8262 & -1.3685 & -0.7916 & -1.4405 & -0.7595 & -1.5277 & -0.7293 & -1.6434 \\
\hline-0.7392 & -1.7245 & -0.7014 & -1.8256 & -0.6666 & -1.8941 & -0.6343 & -1.9473 & -0.6045 & -1.9759 \\
\hline-0.6423 & -1.9743 & -0.6045 & -2.0022 & -0.5698 & -1.9991 & -0.5375 & -1.9931 & -0.5075 & -1.9927 \\
\hline-0.5173 & -2.0707 & -0.4795 & -2.0853 & -0.4448 & -2.0808 & -0.4125 & -2.0746 & -0.3826 & -2.0848 \\
\hline-0.4661 & -2.0603 & -0.4283 & -2.0697 & -0.3936 & -2.0648 & -0.3614 & -2.0586 & -0.3314 & -2.0729 \\
\hline-0.3891 & -1.9598 & -0.3513 & -1.9629 & -0.3166 & -1.9573 & -0.2844 & -1.9511 & -0.2545 & -1.9703 \\
\hline-0.3265 & -1.9303 & -0.2887 & -1.9249 & -0.2539 & -1.9301 & -0.2217 & -1.9431 & -0.1918 & -1.9741 \\
\hline-0.2173 & -1.8858 & -0.1796 & -1.9066 & -0.1448 & -1.9378 & -0.1126 & -1.9751 & -0.0826 & -2.0270 \\
\hline-0.1289 & -1.9105 & -0.0911 & -1.9476 & -0.0563 & -1.9831 & -0.0242 & -2.0182 & 0.0058 & -2.0653 \\
\hline-0.0369 & -1.9652 & 0.0009 & -1.9950 & 0.0357 & -2.0279 & 0.0679 & -2.0676 & 0.0978 & -2.1198 \\
\hline 0.0259 & -2.0171 & 0.0637 & -2.0428 & 0.0984 & -2.0725 & 0.1306 & -2.1095 & 0.1606 & -2.1579 \\
\hline 0.0628 & -2.0475 & 0.1006 & -2.0708 & 0.1353 & -2.0987 & 0.1675 & -2.1341 & 0.1975 & -2.1803 \\
\hline 0.0775 & -2.0597 & 0.1152 & -2.0820 & 0.1500 & -2.1092 & 0.1822 & -2.1439 & 0.2122 & -2.1893 \\
\hline 0.0959 & -2.0749 & 0.1337 & -2.0960 & 0.1684 & -2.1223 & 0.2006 & -2.1562 & 0.2306 & -2.2005 \\
\hline 0.1292 & -2.1024 & 0.1670 & -2.1214 & 0.2017 & -2.1460 & 0.2339 & -2.1784 & 0.2639 & -2.2207 \\
\hline 0.1514 & -2.1207 & 0.1891 & -2.1383 & 0.2239 & -2.1618 & 0.2561 & -2.1932 & 0.2861 & -2.2342 \\
\hline 0.1773 & -2.1421 & 0.2151 & -2.1580 & 0.2498 & -2.1802 & 0.2820 & -2.2105 & 0.3120 & -2.2500 \\
\hline 0.1995 & -2.1604 & 0.2372 & -2.1749 & 0.2720 & -2.1960 & 0.3042 & -2.2253 & 0.3342 & -2.2635 \\
\hline 0.2217 & -2.1840 & 0.2595 & -2.1992 & 0.2942 & -2.2221 & 0.3264 & -2.2540 & 0.3564 & -2.2967 \\
\hline 0.2551 & -2.2231 & 0.2929 & -2.2410 & 0.3276 & -2.2686 & 0.3598 & -2.3071 & 0.3898 & -2.3606 \\
\hline 0.2737 & -2.2448 & 0.3115 & -2.2643 & 0.3462 & -2.2944 & 0.3784 & -2.3367 & 0.4084 & -2.3962 \\
\hline 0.2849 & -2.2580 & 0.3227 & -2.2783 & 0.3574 & -2.3100 & 0.3896 & -2.3545 & 0.4196 & -2.4176 \\
\hline 0.2963 & -2.2713 & 0.3341 & -2.2926 & 0.3688 & -2.3258 & 0.4010 & -2.3726 & 0.4310 & -2.4394 \\
\hline 0.3152 & -2.2934 & 0.3529 & -2.3162 & 0.3877 & -2.3521 & 0.4199 & -2.4026 & 0.4499 & -2.4756 \\
\hline 0.3341 & -2.3283 & 0.3719 & -2.3555 & 0.4066 & -2.3939 & 0.4388 & -2.4430 & 0.4688 & -2.5130 \\
\hline 0.4330 & -2.5581 & 0.4708 & -2.6189 & 0.5056 & -2.6692 & 0.5378 & -2.6935 & 0.5677 & -2.7131 \\
\hline 0.4370 & -2.5672 & 0.4748 & -2.6295 & 0.5095 & -2.6800 & 0.5417 & -2.7035 & 0.5717 & -2.7212 \\
\hline 0.5339 & -2.8253 & 0.5717 & -2.8160 & 0.6064 & -2.8078 & 0.6386 & -2.8014 & 0.6686 & -2.7995 \\
\hline 0.6966 & -2.9057 & 0.7344 & -2.8455 & 0.7692 & -2.7974 & 0.8013 & -2.7638 & 0.8313 & -2.7480 \\
\hline 0.8349 & -3.0277 & 0.8727 & -2.9888 & 0.9075 & -2.9690 & 0.9396 & -2.9771 & 0.9696 & -3.0217 \\
\hline 1.1358 & -3.7761 & 1.1738 & -3.6652 & 1.2084 & -3.5770 & 1.2405 & -3.5305 & 1.2707 & -3.5042 \\
\hline
\end{tabular}

Notes. ${ }^{(a)}$ Photon energies $\epsilon$ are in $\mathrm{eV} .{ }^{(b)}$ Photon densities $\epsilon \frac{\mathrm{d} n_{\gamma}}{\mathrm{d} \epsilon}$ are in $\left[\mathrm{cm}^{-3}\right]$. 
Table 3. Photon proper number density as a function of redshift (3).

\begin{tabular}{|c|c|c|c|c|c|c|c|c|c|}
\hline \multicolumn{2}{|c|}{$z=2.3$} & \multicolumn{2}{|c|}{$z=2.6$} & \multicolumn{2}{|c|}{$z=2.9$} & \multicolumn{2}{|c|}{$z=3.2$} & \multicolumn{2}{|c|}{$z=3.5$} \\
\hline $\log \epsilon^{a}$ & $\log \left(\epsilon \frac{\mathrm{d} n_{\gamma}}{\mathrm{d} \epsilon}\right)^{b}$ & $\log \epsilon$ & $\log \left(\epsilon \frac{\mathrm{d} n_{\gamma}}{\mathrm{d} \epsilon}\right)$ & $\log \epsilon$ & $\log \left(\epsilon \frac{\mathrm{d} n_{\gamma}}{\mathrm{d} \epsilon}\right)$ & $\log \epsilon$ & $\log \left(\epsilon \frac{\mathrm{d} n_{\gamma}}{\mathrm{d} \epsilon}\right)$ & $\log \epsilon$ & $\log \left(\epsilon \frac{\mathrm{d} n_{\gamma}}{\mathrm{d} \epsilon}\right)$ \\
\hline-2.365 & 3.089 & -2.3271 & 3.1650 & -2.2923 & 3.2345 & -2.2602 & 3.2989 & -2.2302 & 3.3588 \\
\hline-2.315 & 2.943 & -2.2771 & 3.0190 & -2.2423 & 3.0885 & -2.2102 & 3.1529 & -2.1802 & 3.2128 \\
\hline-2.265 & 2.762 & -2.2271 & 2.8380 & -2.1923 & 2.9075 & -2.1602 & 2.9719 & -2.1302 & 3.0318 \\
\hline-2.215 & 2.540 & -2.1771 & 2.6160 & -2.1423 & 2.6855 & -2.1102 & 2.7499 & -2.0802 & 2.8098 \\
\hline-2.165 & 2.273 & -2.1271 & 2.3490 & -2.0923 & 2.4185 & -2.0602 & 2.4829 & -2.0302 & 2.5428 \\
\hline-2.115 & 1.955 & -2.0771 & 2.0310 & -2.0423 & 2.1005 & -2.0102 & 2.1649 & -1.9802 & 2.2248 \\
\hline-2.065 & 1.580 & -2.0271 & 1.6560 & -1.9923 & 1.7255 & -1.9602 & 1.7899 & -1.9302 & 1.8498 \\
\hline-2.015 & 1.199 & -1.9771 & 1.2640 & -1.9423 & 1.3252 & -1.9102 & 1.3519 & -1.8802 & 1.4118 \\
\hline-1.931 & 0.5723 & -1.8931 & 0.5938 & -1.8586 & 0.6171 & -1.8602 & 0.9315 & -1.8302 & 0.9724 \\
\hline-1.785 & 0.2097 & -1.7471 & 0.1323 & -1.7124 & 0.0481 & -1.6801 & -0.0512 & -1.6501 & -0.1655 \\
\hline-1.617 & -0.1159 & -1.5795 & -0.2188 & -1.5449 & -0.3124 & -1.5127 & -0.4018 & -1.4827 & -0.4929 \\
\hline-1.533 & -0.3050 & -1.4953 & -0.3991 & -1.4605 & -0.4877 & -1.4283 & -0.5832 & -1.3984 & -0.6975 \\
\hline-1.428 & -0.4735 & -1.3905 & -0.5737 & -1.3558 & -0.6790 & -1.3236 & -0.7865 & -1.2936 & -0.9010 \\
\hline-1.341 & -0.7570 & -1.3034 & -0.8620 & -1.2687 & -0.9610 & -1.2365 & -1.0610 & -1.2065 & -1.1698 \\
\hline-1.262 & -0.9527 & -1.2242 & -1.0576 & -1.1895 & -1.1603 & -1.1573 & -1.2683 & -1.1273 & -1.3898 \\
\hline-1.232 & -0.9314 & -1.1943 & -0.9965 & -1.1595 & -1.0390 & -1.1273 & -1.0687 & -1.0973 & -1.0858 \\
\hline-1.165 & -1.106 & -1.1273 & -1.1875 & -1.0925 & -1.2551 & -1.0604 & -1.3088 & -1.0304 & -1.3549 \\
\hline-0.9888 & -1.260 & -0.9512 & -1.2984 & -0.9165 & -1.2831 & -0.8844 & -1.2378 & -0.8545 & -1.2016 \\
\hline-0.8642 & -1.470 & -0.8262 & -1.5370 & -0.7916 & -1.6996 & -0.7595 & -1.9539 & -0.7293 & -2.2334 \\
\hline-0.7672 & -1.511 & -0.7293 & -1.6757 & -0.6946 & -1.8245 & -0.6625 & -1.9662 & -0.6325 & -2.0691 \\
\hline-0.6880 & -1.797 & -0.6501 & -1.9234 & -0.6155 & -2.0070 & -0.5832 & -2.0617 & -0.5533 & -2.1162 \\
\hline-0.5630 & -2.004 & -0.5252 & -2.0324 & -0.4905 & -2.0593 & -0.4583 & -2.0876 & -0.4283 & -2.1206 \\
\hline-0.4661 & -2.002 & -0.4283 & -2.0170 & -0.3936 & -2.0330 & -0.3614 & -2.0524 & -0.3314 & -2.0771 \\
\hline & -2.121 & -0.3034 & -2.1647 & -0.2687 & -2.2197 & -0.2365 & -2.2707 & -0.2065 & -2.3264 \\
\hline-0.2900 & -2.119 & -0.2523 & -2.1763 & -0.2175 & -2.2504 & -0.1853 & -2.3191 & -0.1553 & -2.3943 \\
\hline-0.2130 & -2.030 & -0.1752 & -2.1046 & -0.1405 & -2.2072 & -0.1083 & -2.3051 & -0.0784 & -2.4165 \\
\hline-0.1503 & -2.050 & -0.1126 & -2.1460 & -0.0778 & -2.2609 & -0.0457 & -2.3652 & -0.0157 & -2.4806 \\
\hline-0.0413 & -2.117 & -0.0035 & -2.2074 & 0.0313 & -2.3161 & 0.0635 & -2.4317 & 0.0934 & -2.5730 \\
\hline 0.0472 & -2.152 & 0.0850 & -2.2468 & 0.1197 & -2.3611 & 0.1519 & -2.4871 & 0.1819 & -2.6348 \\
\hline 0.1392 & -2.210 & 0.1770 & -2.3025 & 0.2118 & -2.4050 & 0.2440 & -2.5218 & 0.2739 & -2.6626 \\
\hline 0.2011 & -2.243 & 0.2398 & -2.3364 & 0.2745 & -2.4489 & 0.3067 & -2.5842 & 0.3367 & -2.7377 \\
\hline 0.2388 & -2.263 & 0.2766 & -2.3563 & 0.3114 & -2.4748 & 0.3436 & -2.6208 & 0.3736 & -2.7819 \\
\hline 0.2535 & -2.271 & 0.2913 & -2.3642 & 0.3261 & -2.4851 & 0.3583 & -2.6354 & 0.3882 & -2.7995 \\
\hline 0.2711 & -2.280 & 0.3098 & -2.3742 & 0.3445 & -2.4979 & 0.3767 & -2.6537 & 0.4067 & -2.8215 \\
\hline 0.3052 & -2.298 & 0.3430 & -2.3921 & 0.3778 & -2.5212 & 0.4100 & -2.6868 & 0.4400 & -2.8614 \\
\hline 0.3274 & -2.310 & 0.3652 & -2.4041 & 0.4000 & -2.5368 & 0.4322 & -2.7089 & 0.4622 & -2.8879 \\
\hline 0.3533 & -2.323 & 0.3911 & -2.4181 & 0.4259 & -2.5549 & 0.4581 & -2.7346 & 0.4881 & -2.9189 \\
\hline 0.3755 & -2.335 & 0.4133 & -2.4301 & 0.4481 & -2.5704 & 0.4803 & -2.7567 & 0.5102 & -2.9455 \\
\hline 0.3977 & -2.377 & 0.4355 & -2.4706 & 0.4703 & -2.6047 & 0.5025 & -2.7811 & 0.5325 & -2.9612 \\
\hline 0.4311 & -2.458 & 0.4690 & -2.5518 & 0.5037 & -2.6693 & 0.5359 & -2.8194 & 0.5659 & -2.9772 \\
\hline 0.4497 & -2.502 & 0.4875 & -2.5969 & 0.5223 & -2.7053 & 0.5545 & -2.8407 & 0.5845 & -2.9861 \\
\hline 0.4609 & -2.529 & 0.4988 & -2.6242 & 0.5335 & -2.7269 & 0.5657 & -2.8536 & 0.5957 & -2.9915 \\
\hline 0.4723 & -2.557 & 0.5101 & -2.6519 & 0.5449 & -2.7490 & 0.5771 & -2.8666 & 0.6071 & -2.9970 \\
\hline 0.4912 & -2.602 & 0.5290 & -2.6978 & 0.5638 & -2.7855 & 0.5960 & -2.8883 & 0.6259 & -3.0060 \\
\hline 0.5101 & -2.635 & 0.5480 & -2.7254 & 0.5827 & -2.8066 & 0.6149 & -2.8999 & 0.6449 & -3.0093 \\
\hline 0.6091 & -2.752 & 0.6469 & -2.8009 & 0.6817 & -2.8586 & 0.7139 & -2.9239 & 0.7438 & -3.0051 \\
\hline 0.6130 & -2.757 & 0.6508 & -2.8039 & 0.6856 & -2.8607 & 0.7178 & -2.9249 & 0.7477 & -3.0049 \\
\hline 0.7099 & -2.804 & 0.7477 & -2.8235 & 0.7825 & -2.8620 & 0.8147 & -2.9216 & 0.8447 & -3.0039 \\
\hline 0.8727 & -2.753 & 0.9105 & -2.7793 & 0.9452 & -2.8259 & 0.9774 & -2.8880 & 1.0073 & -2.9469 \\
\hline 1.011 & -3.155 & 1.0488 & -3.3823 & 1.0835 & -3.8026 & 1.1156 & -4.6210 & 1.1455 & -5.6309 \\
\hline 1.312 & -3.496 & 1.3499 & -3.6416 & 1.3845 & -4.1594 & 1.4168 & -5.1688 & 1.4467 & -6.4232 \\
\hline
\end{tabular}

Notes. ${ }^{(a)}$ Photon energies $\epsilon$ are in eV. ${ }^{(b)}$ Photon densities $\epsilon \frac{\mathrm{d} \eta_{\gamma}}{\mathrm{d} \epsilon}$ are in $\left[\mathrm{cm}^{-3}\right]$. 
Table 4. Photon-photon optical depth as a function of energy and redshift.

\begin{tabular}{|c|c|c|c|c|c|c|c|c|c|c|}
\hline $\begin{array}{l}\text { Energy } \\
{[\mathrm{TeV}]}\end{array}$ & $\begin{array}{c}\tau\left(z, E_{\gamma}\right) \\
z=0.01\end{array}$ & $\begin{array}{c}\tau\left(z, E_{\gamma}\right) \\
z=0.03\end{array}$ & $\begin{array}{l}\tau\left(z, E_{\gamma}\right) \\
z=0.1\end{array}$ & $\begin{array}{l}\tau\left(z, E_{\gamma}\right) \\
z=0.3\end{array}$ & $\begin{array}{l}\tau\left(z, E_{\gamma}\right) \\
z=0.5\end{array}$ & $\begin{array}{l}\tau\left(z, E_{\gamma}\right) \\
z=1.0\end{array}$ & $\begin{array}{l}\tau\left(z, E_{\gamma}\right) \\
z=1.5\end{array}$ & $\begin{array}{l}\tau\left(z, E_{\gamma}\right) \\
z=2.0\end{array}$ & $\begin{array}{l}\tau\left(z, E_{\gamma}\right) \\
z=2.5\end{array}$ & $\begin{array}{l}\tau\left(z, E_{\gamma}\right) \\
z=3.0\end{array}$ \\
\hline 0.00520 & 0.00000 & 0.00000 & 0.00000 & 0.00000 & 0.00000 & 0.00000 & 0.00000 & 0.00001 & 0.00098 & 0.00250 \\
\hline 0.00631 & 0.00000 & 0.00000 & 0.00000 & 0.00000 & 0.00000 & 0.00000 & 0.00000 & 0.00041 & 0.00384 & 0.00706 \\
\hline 0.00767 & 0.00000 & 0.00000 & 0.00000 & 0.00000 & 0.00000 & 0.00000 & 0.00002 & 0.00238 & 0.01031 & 0.01614 \\
\hline 0.00932 & 0.00000 & 0.00000 & 0.00000 & 0.00000 & 0.00000 & 0.00000 & 0.00052 & 0.00770 & 0.02253 & 0.03325 \\
\hline 0.01132 & 0.00000 & 0.00000 & 0.00000 & 0.00000 & 0.00000 & 0.00000 & 0.00269 & 0.01862 & 0.04384 & 0.06308 \\
\hline 0.01375 & 0.00000 & 0.00000 & 0.00000 & 0.00000 & 0.00000 & 0.00020 & 0.00834 & 0.03824 & 0.07863 & 0.10985 \\
\hline 0.01671 & 0.00000 & 0.00000 & 0.00000 & 0.00000 & 0.00000 & 0.00144 & 0.02010 & 0.07046 & 0.13069 & 0.17634 \\
\hline 0.02030 & 0.00000 & 0.00000 & 0.00000 & 0.00000 & 0.00000 & 0.00526 & 0.04148 & 0.11902 & 0.20242 & 0.26437 \\
\hline 0.02466 & 0.00000 & 0.00000 & 0.00000 & 0.00000 & 0.00026 & 0.01411 & 0.07640 & 0.18647 & 0.29553 & 0.37522 \\
\hline 0.02997 & 0.00000 & 0.00000 & 0.00000 & 0.00006 & 0.00168 & 0.03132 & 0.12823 & 0.27492 & 0.41267 & 0.51353 \\
\hline 0.03641 & 0.00000 & 0.00000 & 0.00000 & 0.00075 & 0.00574 & 0.06037 & 0.19977 & 0.38853 & 0.56127 & 0.68797 \\
\hline 0.04423 & 0.00000 & 0.00001 & 0.00013 & 0.00320 & 0.01442 & 0.10498 & 0.29586 & 0.53788 & 0.75593 & 0.91357 \\
\hline 0.05374 & 0.00004 & 0.00015 & 0.00090 & 0.00881 & 0.03012 & 0.17000 & 0.42821 & 0.74227 & 1.01767 & 1.21240 \\
\hline 0.06529 & 0.00017 & 0.00059 & 0.00279 & 0.01897 & 0.05553 & 0.26482 & 0.61799 & 1.02783 & 1.37303 & 1.60979 \\
\hline 0.07932 & 0.00044 & 0.00146 & 0.00625 & 0.03557 & 0.09497 & 0.40704 & 0.89424 & 1.42494 & 1.85166 & 2.13489 \\
\hline 0.09636 & 0.00090 & 0.00292 & 0.01196 & 0.06187 & 0.15657 & 0.62209 & 1.28834 & 1.96569 & 2.48455 & 2.81862 \\
\hline 0.11708 & 0.00164 & 0.00530 & 0.02132 & 0.10376 & 0.25361 & 0.93967 & 1.83323 & 2.68098 & 3.30228 & 3.69168 \\
\hline 0.14224 & 0.00288 & 0.00923 & 0.03649 & 0.17004 & 0.40341 & 1.38927 & 2.56014 & 3.59959 & 4.33198 & 4.78158 \\
\hline 0.17281 & 0.00485 & 0.01543 & 0.06030 & 0.27160 & 0.62492 & 1.99735 & 3.49334 & 4.74466 & 5.59439 & 6.10519 \\
\hline 0.20995 & 0.00785 & 0.02488 & 0.09631 & 0.42039 & 0.93622 & 2.78417 & 4.65017 & 6.12472 & 7.09190 & 7.66666 \\
\hline 0.25507 & 0.01228 & 0.03875 & 0.14856 & 0.62743 & 1.35031 & 3.75962 & 6.02682 & 7.72167 & 8.80131 & 9.44462 \\
\hline 0.30989 & 0.01849 & 0.05813 & 0.22045 & 0.89889 & 1.87116 & 4.91140 & 7.58655 & 9.48565 & 10.6756 & 11.3903 \\
\hline 0.37650 & 0.02671 & 0.08371 & 0.31387 & 1.23409 & 2.49233 & 6.20194 & 9.26186 & 11.3434 & 12.6433 & 13.4434 \\
\hline 0.45742 & 0.03695 & 0.11541 & 0.42716 & 1.62395 & 3.19434 & 7.56774 & 10.9660 & 13.2189 & 14.6472 & 15.5431 \\
\hline 0.55573 & 0.04889 & 0.15213 & 0.55633 & 2.05458 & 3.94206 & 8.93147 & 12.6258 & 15.0605 & 16.6643 & 17.7073 \\
\hline 0.67516 & 0.06195 & 0.19214 & 0.69563 & 2.50232 & 4.69382 & 10.2273 & 14.2003 & 16.9030 & 18.7701 & 20.0347 \\
\hline 0.82027 & 0.07565 & 0.23399 & 0.83870 & 2.94180 & 5.40766 & 11.4154 & 15.7408 & 18.8822 & 21.1241 & 22.7128 \\
\hline 0.99657 & 0.08899 & 0.27442 & 0.97492 & 3.34713 & 6.05113 & 12.5223 & 17.3990 & 21.1526 & 23.9144 & 25.9438 \\
\hline 1.21076 & 0.10138 & 0.31194 & 1.09952 & 3.70442 & 6.61381 & 13.6400 & 19.3428 & 23.8785 & 27.3181 & 29.8841 \\
\hline 1.47098 & 0.11221 & 0.34440 & 1.20615 & 4.00661 & 7.11168 & 14.9226 & 21.7192 & 27.2691 & 31.5636 & 34.8158 \\
\hline & & & & & 7.60245 & & 027 & 31.5446 & 071 & 41.2633 \\
\hline 2.17122 & 0.12901 & 0.39470 & 1.37052 & 4.52208 & 8.16693 & 18.5987 & 28.5221 & 37.1930 & 44.2853 & 50.0201 \\
\hline 2.63787 & 0.13601 & 0.41568 & 1.44294 & 4.82080 & 8.91296 & 21.2999 & 33.6172 & 44.8184 & 54.3879 & 62.3975 \\
\hline 3.20481 & 0.14377 & 0.43949 & 1.53147 & 5.22872 & 9.95027 & 24.9165 & 40.6821 & 55.7377 & 69.2046 & 80.6985 \\
\hline 3.89360 & 0.15419 & 0.47162 & 1.65382 & 5.81803 & 11.3558 & 29.9010 & 50.8562 & 71.9941 & 91.3676 & 108.244 \\
\hline 4.73042 & 0.16911 & 0.51756 & 1.83234 & 6.62927 & 13.2598 & 37.1520 & 66.2602 & 96.9755 & 125.369 & 150.666 \\
\hline 5.74710 & 0.19065 & 0.58408 & 2.08465 & 7.71468 & 15.8888 & 47.9635 & 90.2827 & 135.775 & 177.700 & 218.111 \\
\hline 6.98230 & 0.21876 & 0.67023 & 2.41055 & 9.20728 & 19.6965 & 64.8492 & 128.281 & 195.456 & 257.948 & 338.714 \\
\hline 8.48296 & 0.25607 & 0.78567 & 2.85808 & 11.3604 & 25.5316 & 92.0801 & 187.290 & 285.133 & 384.066 & 621.861 \\
\hline 10.3061 & 0.30752 & 0.94942 & 3.50961 & 14.7030 & 34.7408 & 135.263 & 275.826 & 415.094 & 615.195 & 1451.43 \\
\hline 12.5211 & 0.38586 & 1.19616 & 4.50601 & 20.0272 & 49.6450 & 200.724 & 399.269 & 608.090 & 1175.933 & 3855.73 \\
\hline 15.2122 & 0.51166 & 1.59762 & 6.14335 & 28.6023 & 73.4936 & 293.144 & 563.797 & 952.984 & 2741.434 & 9943.42 \\
\hline 18.4817 & 0.71277 & 2.23537 & 8.72773 & 42.3560 & 109.697 & 414.810 & 786.935 & 1776.48 & 6841.312 & - \\
\hline 22.4539 & 1.03624 & 3.25844 & 12.9010 & 63.5006 & 160.938 & 565.925 & 1156.41 & 3963.61 & - & - \\
\hline 27.2798 & 1.55756 & 4.90160 & 19.4448 & 93.6672 & 227.793 & 749.334 & 2000.75 & 9319.26 & - & - \\
\hline 33.1429 & 2.33730 & 7.33367 & 28.8458 & 133.104 & 308.522 & 1013.70 & 4181.95 & - & - & - \\
\hline 40.2661 & 3.40014 & 10.6261 & 41.1443 & 180.335 & 400.080 & 1548.18 & 9404.14 & - & - & - \\
\hline 48.9203 & 4.72388 & 14.6948 & 55.8835 & 233.223 & 505.738 & 2907.47 & - & - & - & - \\
\hline 59.4344 & 6.23960 & 19.3110 & 72.1970 & 290.373 & 660.765 & 6283.00 & - & - & - & - \\
\hline 72.2083 & 7.82189 & 24.1678 & 89.2541 & 364.018 & 1014.58 & - & - & - & - & - \\
\hline 87.7276 & 9.50683 & 29.3591 & 109.080 & 522.730 & 1997.66 & - & - & - & - & - \\
\hline 106.582 & 11.8962 & 37.2086 & 147.522 & 996.470 & 4510.25 & - & - & - & - & - \\
\hline 129.489 & 18.3001 & 59.3783 & 268.848 & 2318.77 & 9990.42 & - & - & - & - & - \\
\hline 157.319 & 39.6796 & 132.463 & 642.223 & 5410.71 & - & - & - & - & - & - \\
\hline 191.131 & 99.7780 & 333.153 & 1579.26 & - & - & - & - & - & - & - \\
\hline 232.210 & 235.983 & 777.945 & 3498.49 & - & - & - & - & - & - & - \\
\hline
\end{tabular}

Notes. The absorption multiplicative factor is $\mathrm{e}^{-\tau\left(z, E_{\gamma}\right)}$. 